\title{
高温高压下络合物研究的地球 化学意义与实验方法
}

王玉荣

(中国科学院贵阳地球化学研究所)

元素在地壳中怎样迁移和它迁移的形式是什么, 地球化学家们很早就注意探索这个问题 了, 并且做过大量假设. 近二十年来不少学者将络合物的概念逐渐引入地球化学, 作为元素迁 移形式的最为有利的方式,并进行了一系列实验和热力学计算 ${ }^{[1-9]}$.

在国内, 司幼东 ${ }^{[10]}$ 首先将络合物概念引进地球化学. 又在常温常压下,对铍的迁移形式和 沉淀条件进行了研究 ${ }^{[11]}$. 此后, 我们曾对锡 ${ }^{[12]}$ 、铌、钽络合物进行了高温高压实验研究. 在从 事这项工作中, 我们认识到地质过程是多组分复杂体系的化学演变. 因此, 必须借助于络合物 来研究元紊的迁移形式,才能获得令人满意的结果.

\section{一、高温高压下络合物的特性}

高温高压下也存在这样一类化合物, 它们在矿物中或在流体相中有络合的阳离子、阴离子 或电中性分子存在, 而这些络合了的集团, 在溶液中相当稳定, 不易离解为它的组成部分, 这 样一类化合物称之为络合物. 天然过程的高温高压状态, 由于组分复杂、密度大、挥发性组分 浓度高等特点, 利于络合物的形成. 高温高压下络合物的组成大都属配位型络合物和多酸型 络合物. 在自然界中绝大多数化合物是按络合物形态而存在的 ${ }^{[33]}$. 我们可以把矿物中的络 离子团(如 $\mathrm{CO}_{3}^{2-} 、 \mathrm{SO}_{4}^{2-} 、 \mathrm{WO}_{4}^{2-} 、 \mathrm{BO}_{3}^{3-} 、 \mathrm{SiO}_{4}^{4-} \cdots$ )看成是络合物在流体相中一种形式，在物理化 学条件改变时又转化为另一种形式的结果. 高温高压下, 我们主要研究了络合物以下几方面 的特性.

（1）络合物形成体对不同挥发性组分,有不同的选择作用特性: Ahrland ${ }^{[14]}$ 曾将周期表中 的元䇣分为两大类络合物形成体，第一类形成体与 $\mathrm{C} 、 \mathrm{O} 、 \mathrm{~F}$ 形成比较稳定的络合物; 第二类与 $\mathrm{P} 、 \mathrm{~S} 、 \mathrm{Cl}$ 形成比较稳定的络合物; 还有边缘形成体属中间类型(图 1). Щербина ${ }^{[151}$ 曾根据元素 在矿床中分布及共生关系, 来判断元素可能的迁移形式, 并将周期表中元素划分为亲 $\mathrm{F}$ 和亲 $\mathrm{Cl}$ 元素,与图 1 有些相似. 然而, 高温高压下各种形成体对配位体的选择特性, 还需要大量实验 来确定.

外界阳离子, 由于它们之间离子半径和电荷不等, 对络合物稳定性也发生影响, 在高温高 压下显得尤为突出, 表现为各种不同盐类络合物溶解度, 挥发性和热稳定各有差异

本文 1978 年 1 月 24 日收到。

$\cdot 682$. 


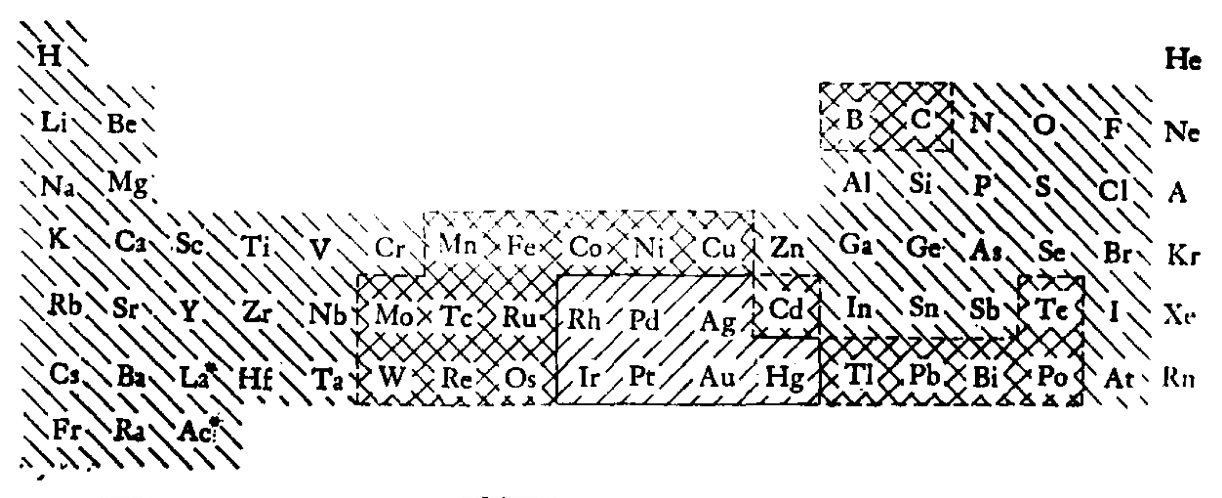

第一类形成体 D7 第二类形成体 边缘形成体

图 1 各种类型形成体在周期表上的分布

（2）络合物的温、压稳定性: 即研究络合物处于各种相态时, 温度和压力是否影响络合物 的分解. 无机化学的成果告诉我们, 许多复盐络合物热稳定性是很大的, 如 $\mathrm{K}_{2} \mathrm{BeF}_{4}$ 的熔点是 $791 \pm 5^{\circ} \mathrm{C} ; \mathrm{K}_{2} \mathrm{TaF}_{7}$ 的熔点是 $730^{\circ} \mathrm{C}$, 而它们熔化时并不分解. 一般说来, 温度增高促进络合 物分解, 压力增大促进络合物稳定.

（3）络合物的离解特性: 即当络合物溶于水后, 在一定温度、压力条件下发生离解或逐级 离解为它的组成部分. 这一般用络合物的不稳定常数或稳定常数来表示.

（4）络合物水解特性: 即络合物溶于水后是否容易进行酸或碱离解. 水是自然界中重要 的组分. 高温高压下的水解作用比常温常压下显得更为普遍和剧烈, 所以这个特性的研究尤 为重要. 水解过程是由络离子在溶液中首先发生离解后, 水与中心原子相结合, 发生极化作 用,水分子进行离解,其过程用以下反应式表示:

$$
\begin{gathered}
\mathrm{SnF}_{6}^{2-}=\mathrm{SnF}_{5}^{-}+\mathrm{F}^{-} ; \mathrm{SnF}_{5}^{-}+\mathrm{H}_{2} \mathrm{O}=\left[\mathrm{SnF}_{5} \cdot \mathrm{H}_{2} \mathrm{O}\right]^{-} ; \\
{\left[\mathrm{SnF}_{5} \cdot \mathrm{H}_{2} \mathrm{O}\right]^{-}=\left[\mathrm{SnF}_{5} \cdot \mathrm{OH}\right]^{2-}+\mathrm{H}^{+} .}
\end{gathered}
$$

以上反应加合后得:

$$
\mathrm{SnF}_{6}^{2-}+\mathrm{H}_{2} \mathrm{O}=\left[\mathrm{SnF}_{5} \cdot \mathrm{OH}\right]^{2-}+\mathrm{HF},
$$

这样逐级水解. 在高温高压作用下, $\mathrm{OH}^{-}$基发生脱水反应得:

$$
\mathrm{SnF}_{6}^{2-}+2 \mathrm{H}_{2} \mathrm{O}=\mathrm{SnO}_{2} \downarrow+2 \mathrm{~F}^{-}+4 \mathrm{HF},
$$

设 $\mathrm{H}_{2} \mathrm{O}$ 和 $\mathrm{SnO}_{2}$ 的活度等于 1 ,

$$
\text { 水解常数 } K=\frac{\left[\mathrm{F}^{-}\right]^{2}[\mathrm{HF}]^{4}}{\left[\mathrm{SnF}_{6}^{2-}\right]} \text {. }
$$

因此,在一定温度下, 水解与溶液中 $\mathrm{F}^{-}$活度及酸度有关, 并也表明压力增大促进络合物稳 定. 根据我们的实验结果表明, 水解与温度的关系有这样规律, 从低温到高温水解作用逐渐增 大, 在临界温度附近有个最大值, 在超临界状态下, 随着温度增高络合物水解作用降低反而趋 于稳定(图 2、3). 这种定量关系还有待进一步研究.

（5）络合物氧化还原稳定性：当元素形成络合物以后，它的氧化还原电位也随之发生变 化. 一般情况可趋使它的价态更稳定, 这个特性, 对于地质过程中的变价元素的研究十分重 要. 随温度增高, 变价元素中的低价状态更稳定. 铁元素就是一个明显例子, 常温常压下溶液 中 $\mathrm{Fe}^{3+}$ 很稳定, $200^{\circ} \mathrm{C}$ 高温下主要以 $\mathrm{FeCl}^{+}$存在. 


\section{二、络合物研究的地球化学意义}

根据络合物本身的特性和地质情况, 我们认为络合物研究有以下几个方面的意义.

(1) 络合物研究对了解元索成矿的物理化学条件和探讨成矿过程的机理是十分重要的。 这首先涉及到各种挥发性组分和碱金属对成矿元紊的迁移作用. 如我们实验结果证明, F 对 $\mathrm{Sn} 、 \mathrm{Nb} ， \mathrm{Ta}$ 投运能力都很强. 还证明 $\mathrm{Li} 、 \mathrm{Na} 、 \mathrm{~K}$ 对 $\mathrm{Nb} 、 \mathrm{Ta}$ 気络合物投运能力的影响也不相 同, $\mathrm{Li}$ 和 $\mathrm{Na}$ 可提高 $\mathrm{Ta}$ 氟络合物的搬运能力, 而与 $\mathrm{K}$ 相比, 对 $\mathrm{Nb}$ 影响不大. 这都属于迁移形 式的组成问题. 研究络合物的稳定也就是研究不同温度、压力、 $\mathrm{pH} 、 \mathrm{Eh} 、$ 组分浓度等各种条件 下，元系迁移、沉淀、富集的条件. 当元素富集成矿时，应有这样的过程, 即首先是碱金属破 坏, 然后是络合物内界挥发性组分破坏, 元素才能完全沉淀下来. БарсукоB ${ }^{[16]}$ 长期以来研究锡 矿床, 并且进行了实验研究. 他采用测 $\mathrm{F}$ 的地球化学晕的办法来寻找盲矿体, 也还十分见效. 这和我们在南岭花岗岩地区一样, 当我们遇到钠化和云英岩化时, 就有可能找到稀有元素矿 床.

（2）因为络合物的生成具有较高的选择性能, 并使每个元素的性质发生变化. 所以对于 研究地壳中化学性质很相似的元素如 $\mathrm{Nb}$ 与 $\mathrm{Ta}, \mathrm{Zr}$ 与 Hf, 以及稀土元素等在地球化学演化过 程中的分离与分配是十分有利的. 我们实验证明, 在超临界高温气热溶液中, $\mathrm{Ta}$ 搬运能力比 $\mathrm{Nb}$ 大, 所以 $\mathrm{Ta}$ 富集在岩体的顶部或边部, 而发生 $\mathrm{Nb}$ 和 $\mathrm{Ta}$ 的分离.

（3）由于络合物溶解度大, 稳定性高等特点, 用来解释再造一类矿床也是十分有利的. 因 为各种成因的热液在它运动的通道上与围岩相互作用,将围岩中的成矿元系淋滤出来, 经迁移 后再富集成矿的这一过程中, 如果成矿元素形成络合物, 那么对于解释成矿元紊的淋滤、迁移 以及它们再沉腚、富集、成矿的过程机理是十分必要的.

（4）各种元紊的络合物, 它们之间稳定性的差异可以通过实验测定来比较的,因此当我们 研究一组元索的迁移形式和它们稳定的物理化学特征时, 就可以根据它们之间稳定性大小, 来 判断这组成矿元素分异的序列和构成元素分带分布情况. 这种元素分异的规律研究, 将为隐 伏矿体的寻找和目前迅速发展起来的元素地球化学数理统计提供理论依据.

（5）研究元素在熔体、液体、气体的各种相态中的迁移形式和稳定条件, 以及它们在各相 态之间分配的系数大小. 就可以探讨成矿元素的物质来源、矿床成因, 并提供在什么条件下有 可能形成富矿体.

(6) 元素在地壳中的演化过程, 是一个复杂的多组分体系的变化. 当我们研究了每种元 素迁移形式的物理化学特性后, 并求得成矿元素与造岩元素之间的互相关系, 那么就能探索元 素在地売中演化、分类的内在原因.

\section{三、高瑥高压下研究络合物的方法}

常温常压下研究络合物的方法很多, 然而在高温高压条件下, 就受到很多限制. 我们只能 根据目前条件,介绍一下我们研究这个工作所采用的方法. 目前我们仅用莫雷式小高压、莫雷 式 $\Pi$ 型高压金及 Tuttle 型高压釜三种. 都是封闭的用水产生压力的间断式操作.

（1）溶解度法: 这是最经典的方法, 但仍是最可靠的方法之一. 它的主要论据是建立在 
微溶化合物的溶解度. 由于络合物的形成而增大. Баранова ${ }^{[17]}$ 用此种方法研究了 $\mathrm{PbCO}_{3}$ 在 $\mathrm{Na}_{2} \mathrm{CO}_{3}$ 溶液中, 不同温度 $\left(200-250^{\circ} \mathrm{C}\right)$ 时络合物的组成和它们的稳定常数. 目前国外大都 用这种方法.

（2）水解测定法: 是将络合物溶于水后, 测定它的酸碱变化,和在各种物理化学条件下到 达平衡后, 测定它水解了多少. Beyc ${ }^{[18]}$ 曾认为络合物在高温下强烈水解, 是它能否作为元来 搬运形式的最大威胁. 他虽根据水的特性也估计到络合物在超临界高温下应该稳定，但没有 进行这方面的实验研究. 与此同时, 我们研究锡的搬运形式时, 也发现络合物水解作用很强 烈, 水解生成了 $\mathrm{SnO}_{2}$ 的沉淀物, 沉淀物经淬火到室温后, 过夜, 也没有返回到溶液中. 这样, 我 们就采用固液分离, 定量测定了锡络合物的水解性能与温度的关系. 求得了水解最大值在水 的临界温度附近. 超临界高温条件下随温度增高, 络合物更趋于稳定 ${ }^{[12]}$. 这以后, 我们用同样 的方法又测定了 $\mathrm{Nb} 、 \mathrm{Ta}$ 氧络合物水解与温度的关系, 也得到了同样的规律 (图 2、3). ArekcaдpoB ${ }^{[19]}$ 用溶解度法研究 Nb、 $\mathrm{Ta}$ 络合物时, 得到了和我们相似的曲线 (图 4). 他把 300-

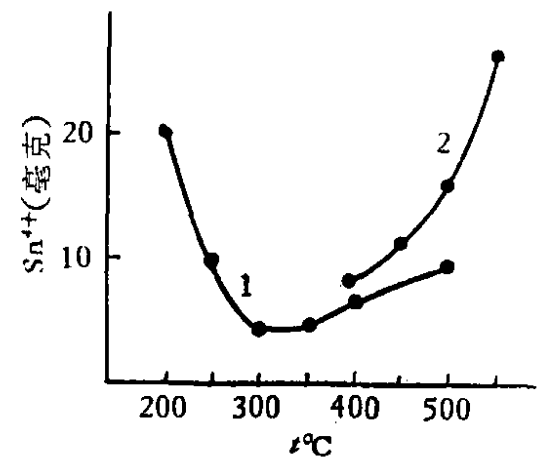

图 $2 \mathrm{Na}_{2} \mathrm{SnF}_{6}$ 的水解与温度的关系

填充度 $53.2 \%$

1. $\mathrm{Na}_{2} \mathrm{SnF}_{6}+\mathrm{H}_{2} \mathrm{O}$;

2. $\mathrm{Na}_{2} \mathrm{SnF}_{6}+\mathrm{K}_{2} \mathrm{SiF}_{6}+\mathrm{H}_{2} \mathrm{O}$

$400{ }^{\circ} \mathrm{C}$ 出现含量降低的现象，称之为“门坎”。 其实,在我们看来, 这“门坎”是由于水解作用 造成的,而不是别的原因.

水解法只适用于这样一类元素的络合物 研究, 即高温高压下水解平衡后, 淬火到室 温, 水解产物不易返回到水解后的母液中. 这 样, 迅速分离固液两相进行分析测定, 就能代 表原来高温高压下固液平衡的状况，经我们 实验表明, $\mathrm{Sn}^{4+} 、 \mathrm{Nb}^{5+} 、 \mathrm{Ta}^{5+} 、 \mathrm{Fe}^{3+}$ 都具有此 种特性. 估计 $\mathrm{Ti} 、 \mathrm{Zr} 、 \mathrm{Hf} 、 \mathrm{Al} 、 \mathrm{Ga} 、 \mathrm{Sb}$ 等两性 元素也都有类似性质. 这在化学中解释为, 水解后的产物易发生老化，性质变得不活泼， 溶解度很小. 对于这一类元素，用水解法要

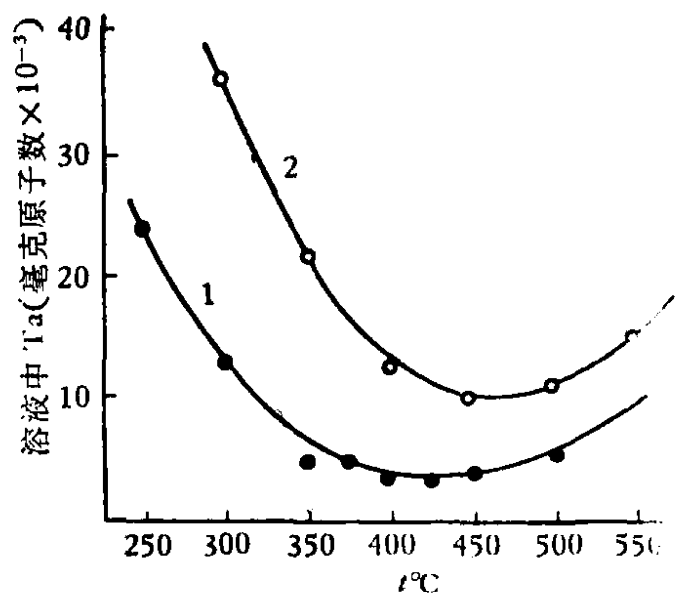

图 $3 \mathrm{~K}_{2} \mathrm{TaF}$ 水解与温度(低力)的关系 填充度 $53.2 \%$

1. $\mathrm{K}_{2} \mathrm{TaF},+\mathrm{H}_{2} \mathrm{O}$;

2. $\mathrm{K}_{2} \mathrm{TaF}_{7}+\mathrm{Na}_{2} \mathrm{SiF}_{6}+\mathrm{H}_{2} \mathrm{O}$

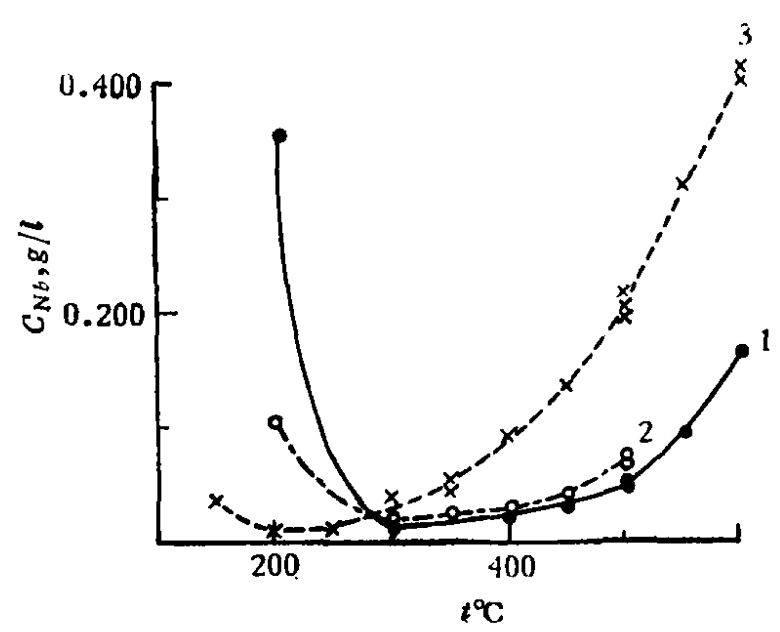

图 4 不同温度下铌在各种溶液中浓度的变化 1. $0.75 \mathrm{MKHCO}_{3}+2.25 \mathrm{MKCl}$;

2. $0.75 \mathrm{KHCO}_{3}+0.5 \mathrm{MKF}+1.75 \mathrm{MKCl}$ :

3. $0.75 \mathrm{MKHCO}_{3}+0.5 \mathrm{MK}_{2} \mathrm{HPO}_{4}+0.75 \mathrm{MKCl}$ 
比用溶解度法, 更容易达到平衡.

（3）气相搬运反应法: 是在 П 型金中两个上部相通的反应室内进行. 这是我们企图定 量测定气液平衡, 和超临界状态下流体相中物质的存在状况而设计的. 利用分子在高温下自 由扩散达到平衡, 经淬火保留流体相组分, 这行分析测定, 而免去复杂的取样装置. 经实验证 明, 这个简便的方法,对于研究那些易挥发的元素化合物及研究超临界状态下物质之间的平衡 是有效的. 我们曾用此种方法在超临界状态下比较 $\mathrm{Nb}$ 和 $\mathrm{Ta}$ 氧络合物迁移能力的大小, 以及 在水的临界温度以下发现 $\mathrm{FeCl}_{2}$ 具有较大的挥发性.

（4）分配法 ${ }^{[20]}$ : 在 Turtle 型高压釜中进行. 将岩石矿物或它们的玻璃和挥发性及碱金 属组分等一起封闭在 $\phi_{5}-\phi_{7} \mathrm{~m} / \mathrm{m}$ 的小金管内, 经高温高压作用平衡后, 迅速冷却尽量保存平衡 时的状况, 测定成矿元素和造岩元素在各种相态中（结晶相一玻璃相一水相）的分配和相关系 数. 这对了解元素从一种相态转变为另一相态时, 各种碱金属和挥发性组分, 对元素迁移状况 的影响,是很重要的.

实验过程中, 还涉及到络合物的制备、样品的老化、平衡的测定、临界温度的估计，以及 $\mathrm{pH} 、 \mathrm{Eh}$ 条件的控制、防止腐蚀、取样分析等一系列实验技术和方法. 这些在实验过程中都不 能忽视.

高温高压下络合物实验研究方法还有待进一步探索和建立，对所测定数据进行处理也有 待进一步研究. 以上仅是初步工作的总结.

致谢：本工作曾得到司幼东、涂光炽、郭承基等教授的指导,并得到王道德、李加田、梁伟仪、卢家烂、森 文苓等同志的帮助,特此表示感谢.

[1] Crerar, A. David \& Barnes, H. L., Econ. Geol., 71 (1976), 4, 772-792.

[:] Helgeson, H. C., Complexing and Hydrothermal Ore Deposition, London. 1964, 1-15.

[3] Helgeson, H. C., Amer. J. of Sci., 267 (1969), 7, 729-807.

[4] Щербпня В. В., Вопрось иеохимии и жинерапоик. Ивд. АН. СССР, 1956, $72-82$

[5] Берс A. A., leoxuxus, 1958, 4, 307-313.

[6] Барсуков В. Л., Доки. AH СCCP, Нов., 93 (1953), 6, 1065-1068.

[ 7 ] Иванова Г. Ф. и Ходаеовскии И. Л., Геохииия, 1968, 8, 930-939.

[8] Кувнецв В. А., Гео. Руа. Месторохд., 1969. 4,, 114-117.

[ 9 ] Папутин Р. П. и Сободев В. П., ТР. ИМГРЯ, 18 (1963), 44-52.

[10] 司幼东, 地质科学, $1963,1,19-28$.

[11] 司幼东、黄释华, 地质科学, 1963, 4, 169-176.

[12] 王玉荣等, 地质科学, 1965, 2, 141-154.

[13] 严志弦, 络合物化学, 人民数育出版社, 1960, 8-9.

[14] Ahrland, S., Chatt, J. \& Davies, N. R., Quarterly Reviews, 12 (1958), 265-276.

[15] Щер6вна В. В., Геохижик, 1963, 8, 721-724.

[16] Барсуков В. Л., Основнъе черть геохихии олова, Изд. Наука, Мос., 1974, $130-141$.

[17] Баравова Н. H., Геохимия, 1968, 1, 17-25.

[18] Бөус А. А. п Собхев Б. П., Проблежъ иежезиса руд, Межд. Геодо. Конгресс ХХІІ Сесея, Изд. Недра, $1964,36-49$.

[19] Алексадров И. В., Модели әидогеного тантило-ниобиевого оруденения. Изд. Наука, Мос. 1973, 4546. $138-140$.

¡20† Holland, H. D., Econ. Geol., 67 (1972), 3, 281-201. 